\title{
Oral Immunotherapy for Treatment of Egg Allergy in Children
}

\author{
A. Wesley Burks, M.D., Stacie M. Jones, M.D., Robert A. Wood, M.D., David M. Fleischer, \\ M.D., Scott H. Sicherer, M.D., Robert W. Lindblad, M.D., Donald Stablein, Ph.D., Alice K. \\ Henning, M.S., Brian P. Vickery, M.D., Andrew H. Liu, M.D., Amy M. Scurlock, M.D., Wayne \\ G. Shreffler, M.D., Ph.D., Marshall Plaut, M.D., Hugh A. Sampson, M.D., and for the \\ Consortium of Food Allergy Research (CoFAR) \\ Department of Pediatrics, Duke University Medical Center, Durham, NC (A.W.B., B.P.V.); the \\ Department of Pediatrics, University of Arkansas for Medical Sciences and Arkansas Children's \\ Hospital, Little Rock (S.M.J., A.M.S.); the Department of Pediatrics, Johns Hopkins University \\ Medical Center, Baltimore (R.A.W.); the Department of Pediatrics, National Jewish Health, \\ Denver (D.M.F., A.H.L.); the Department of Pediatrics, Mount Sinai School of Medicine, New York \\ (S.H.S., H.A.S.); EMMES, Rockville (R.W.L., D.S., A.K.H.), and the National Institutes of Health, \\ Bethesda (M.P.) - both in Maryland; and the Department of Pediatrics, Massachusetts General \\ Hospital, Harvard Medical School, Boston (W.G.S.).
}

\section{Abstract \\ BACKGROUND—For egg allergy, dietary avoidance is the only currently approved treatment. We evaluated oral immunotherapy using egg-white powder for the treatment of children with egg allergy.}

METHODS-In this double-blind, randomized, placebo-controlled study, 55 children, 5 to 11 years of age, with egg allergy received oral immunotherapy (40 children) or placebo (15). Initial dose-escalation, build-up, and maintenance phases were followed by an oral food challenge with egg-white powder at 10 months and at 22 months. Children who successfully passed the challenge at 22 months discontinued oral immunotherapy and avoided all egg consumption for 4 to 6 weeks. At 24 months, these children underwent an oral food challenge with egg-white powder and a cooked egg to test for sustained unresponsiveness. Children who passed this challenge at 24 months were placed on a diet with ad libitum egg consumption and were evaluated for continuation of sustained unresponsiveness at 30 months and 36 months.

RESULTS-After 10 months of therapy, none of the children who received placebo and 55\% of those who received oral immunotherapy passed the oral food challenge and were considered to be desensitized; after 22 months, $75 \%$ of children in the oral-immunotherapy group were desensitized. In the oral-immunotherapy group, $28 \%$ (11 of 40 children) passed the oral food challenge at 24 months and were considered to have sustained unresponsiveness. At 30 months and 36 months, all children who had passed the oral food challenge at 24 months were consuming egg. Of the immune markers measured, small wheal diameters on skin-prick testing and increases in egg-specific IgG4 antibody levels were associated with passing the oral food challenge at 24 months.

CONCLUSIONS-These results show that oral immunotherapy can desensitize a high proportion of children with egg allergy and induce sustained unresponsiveness in a clinically

Copyright @ 2012 Massachusetts Medical Society.

Address reprint requests to Dr. Burks at the University of North Carolina, Department of Pediatrics, 260 MacNider, Bldg., Campus

Box 7220, Chapel Hill, NC, 27599-7220, or at wburks@email.unc.edu.

Drs. Burks and Jones contributed equally to this article.

Disclosure forms provided by the authors are available with the full text of this article at NEJM.org. 
significant subset. (Funded by the National Institutes of Health; ClinicalTrials.gov number, NCT00461097.)

In the United States, $4 \%$ of children have a food allergy, ${ }^{1}$ which affects health and quality of life. ${ }^{2}$ Egg allergy has a cumulative prevalence of approximately $2.6 \%$ by 2.5 years of age, ${ }^{3}$ with allergic reactions varying in severity from mild urticaria to systemic anaphylaxis. Severe allergic reactions can occur with a single bite of cooked egg (approximately $70 \mathrm{mg}$ of egg protein). Children with egg allergy are placed on egg-free diets, but total avoidance of egg is difficult. Avoidance places a constant responsibility on patients and caregivers, leaves patients vulnerable to unintentional ingestion and anaphylaxis, and influences quality of life. $^{4,5}$

Given these challenges, new treatment strategies are being explored. The goal of allergen immunotherapy is to produce a more sustained clinical effect than desensitization, including immune tolerance (i.e., long-term loss of allergic reactivity after discontinuation of therapy). Desensitization, a state in which the threshold dose of food that triggers an allergic reaction is raised during therapy, is more easily achieved. Traditional subcutaneous immunotherapy, which is effective against certain aeroallergens, ${ }^{6,7}$ is unsafe for the treatment of food allergy. ${ }^{8,9}$ Oral immunotherapy appears to be safer than subcutaneous immunotherapy for food allergens and induces desensitization. Oral immunotherapy has been successful in desensitizing patients to several food allergens in small clinical trials, most of which were not controlled. ${ }^{10-20}$ In the current study, we did not study the induction of immune tolerance, but we assessed what we call "sustained unresponsiveness," defined as the ability, after 22 months of oral immunotherapy and subsequent avoidance of egg consumption for 4 to 6 weeks, to consume $10 \mathrm{~g}$ of egg-white powder and a whole cooked egg without clinically significant symptoms. In addition, children who passed the oral food challenge at 24 months were placed on an ad libitum diet and followed for 12 more months.

We conducted a multicenter, double-blind, randomized, placebo-controlled study of the effectiveness and safety of oral immunotherapy, including its capacity to induce sustained unresponsiveness, in children with egg allergy.

\section{METHODS}

\section{STUDY DESIGN AND PARTICIPANT SELECTION}

The primary end point of the study was the induction of sustained unresponsiveness after 22 months of oral immunotherapy with egg. Secondary end points included desensitization, which was defined as the ability to pass an oral food challenge with $5 \mathrm{~g}$ of egg-white powder at 10 months and with $10 \mathrm{~g}$ at 22 months, while still receiving daily oral immunotherapy, and the safety of oral immunotherapy. The study protocol is available with the full text of this article at NEJM.org.

Eligible participants were 5 to 18 years of age and had a convincing clinical history of egg allergy (shown by the development of allergic symptoms within minutes to 2 hours after ingesting egg) and a serum egg-specific IgE antibody level of more than $5 \mathrm{kU}$ per liter for children 6 years of age or older, or $12 \mathrm{kU}$ per liter or more for those 5 years old. These levels were chosen to exclude children who were likely to outgrow the allergy during the course of the study. ${ }^{21,22}$ Children with a history of severe anaphylaxis (i.e., previous hypotension) after egg consumption were excluded.

\section{STUDY OVERSIGHT}

The study protocol and consent forms were approved by the institutional review board at each clinical site. The study was conducted under an investigational new drug application to 
the Food and Drug Administration and was monitored by an independent data and safety monitoring board from the National Institute of Allergy and Infectious Diseases. Written informed consent was obtained from parents or guardians, with assent from children older than 7 years of age.

The authors attest to the veracity and completeness of the data and analyses as well as to the fidelity of the study to the protocol. Raw egg-white powder was purchased from a commercial manufacturer (Deb-El Food Products). Immune-testing reagents were provided at a discounted rate by Greer Laboratories and Phadia.

\section{RANDOMIZATION AND DOSING}

The participants were randomly assigned by means of a centralized computer algorithm to receive either double-blind oral immunotherapy with egg or placebo (in a ratio of 8:3) at five clinical sites (with a total of 40 children receiving oral immunotherapy, and 15 placebo). The study was blinded through the first oral food challenge at 10 months (Fig. 1). Thereafter, placebo was stopped, and the children in the placebo group were followed through 24 months, whereas treatment was continued in the oral-immunotherapy group on an open-label basis. Egg-white powder and matching placebo (cornstarch) were weighed and put into vials at a central pharmacy and then distributed to the pharmacies at the study sites.

\section{ORAL-IMMUNOTHERAPY PROTOCOL}

The protocol for oral immunotherapy consisted of three phases: an initial-day dose escalation, a build-up phase, and a maintenance phase during which participants ingested up to $2 \mathrm{~g}$ of egg-white powder per day, which is the approximate equivalent of one third of an egg (see the Supplementary Appendix, available at NEJM.org). The children and their families were instructed that the children should avoid egg consumption other than the oral immunotherapy. The severity of allergic reactions was reported with the use of a customized grading system, with scores ranging from 1 (transient or mild discomfort) to 5 (death) (Table S1 in the Supplementary Appendix).

\section{ORAL FOOD CHALLENGE AND FOLLOW-UP}

At 10 months, all participants underwent an oral food challenge consisting of $5 \mathrm{~g}$ (cumulative dose) of egg-white powder. Participants who passed (i.e., consumed who received the placebo were given a subsequent oral food challenge only if the egg-specific the entire amount without having clinically significant allergic symptoms) were considered to be desensitized. Children IgE antibody level was less than $2 \mathrm{kU}$ per liter - a cutoff defined on the basis of risks associated with oral food challenges in children who did not pass an oral food challenge during the previous year and the association of not passing an oral food challenge with elevated levels of egg-specific IgE antibody. ${ }^{21,23}$ Children who received oral immunotherapy underwent a second oral food challenge, with a dose of $10 \mathrm{~g}$ of egg-white powder, at 22 months. The children who passed the oral food challenge at 22 months discontinued oral immunotherapy and avoided any egg consumption for 4 to 6 weeks. At 24 months, these children were given an oral food challenge of $10 \mathrm{~g}$ of egg-white powder, followed 1 hour later by feeding of a whole cooked egg.

The oral food challenge was scored as pass (consumption of the total dose of egg with no clinically significant allergic symptoms) or fail (inability to consume the total dose because of persistent allergic symptoms such as hives, wheezing, vomiting, or laryngeal edema). The scorer was unaware of the study assignments through 10 months. Participants who passed the oral food challenge at 24 months and consumed the whole cooked egg were instructed to 
add egg to their diet ad libitum and to report any adverse events. Egg consumption and adverse events were ascertained by telephone or at clinic visits at 30 months and 36 months.

\section{IMMUNE MARKERS}

Skin-prick testing with egg extract (Greer Laboratories) and saline and histamine controls was performed at enrollment and at 10 months and 22 months. Basophil activation was measured according to CD63 up-regulation on flow cytometry. ${ }^{24}$ Serum egg-specific IgE and IgG4 antibody levels were measured with the use of the Immuno-CAP 100 (Thermo Fisher Scientific).

\section{STATISTICAL ANALYSIS}

We calculated that a sample of 55 participants (40 receiving oral immunotherapy, and 15 placebo) would provide $84 \%$ power, at a two-sided alpha level of 0.05 , to detect a significant between-group difference in the rate of sustained unresponsiveness, assuming an estimated $10 \%$ rate in the placebo group and an estimated $50 \%$ rate in the oralimmunotherapy group. All clinical outcomes were assessed by intention-to-treat analysis. Rates of sustained unresponsiveness were tested with Barnard's test, and an exact confidence interval for the between-group difference in the response rate was calculated. The Wilcoxon rank-sum test was used to evaluate between-group differences in changes from baseline in skin-prick test results (wheal size) and immunoglobulin levels. Basophil activation and immunoglobulin levels were evaluated in repeated-measurement models, with the baseline value as a covariate and unstructured within-person covariance. Logistic regression was used to evaluate the association of selected immune variables with clinical outcomes All analyses were performed with the use of SAS software, version 9.2 (SAS Institute), and StatXact software, version 6 (Cytel Software).

\section{RESULTS \\ STUDY PARTICIPANTS}

A total of 55 participants (11 per institution), with a median age of 7 years, were enrolled; 15 received placebo, and 40 oral immunotherapy with egg (Fig. 1 and Table 1). Of these participants, $91 \%$ reported at least one additional food allergy.

\section{ASSESSMENT OF CLINICAL RESPONSES}

None of the 15 children who received placebo and 22 of the 40 (55\%) who received oral immunotherapy passed the oral food challenge of $5 \mathrm{~g}$ of egg-white powder at 10 months (95\% confidence interval for the difference in the response rate, 30 to $71 \%$; $\mathrm{P}<0.001$ ) (Table 2). Six additional children in the oral-immunotherapy group consumed the cumulative 5-g dose but had clinically significant allergic symptoms at the last dose, and the challenge was deemed a failure in these children. Of the 22 children who passed, 14 had no symptoms, 7 had mild symptoms that resolved without treatment, and 1 had a moderate symptom (throat discomfort) at the fourth dose that resolved without treatment. The median cumulative dose successfully consumed during the oral food challenge at 10 months was $5.00 \mathrm{~g}$ (range, 0.25 to 5.00) in the oral-immunotherapy group versus $0.05 \mathrm{~g}$ (range, 0.00 to 2.75 ) in the placebo group $(\mathrm{P}<0.001)$.

One child who received placebo had an egg-specific IgE antibody level of less than $2 \mathrm{kU}$ per liter and underwent the oral food challenge of $10 \mathrm{~g}$ of egg-white powder at 22 months; the child did not pass this challenge. At 22 months, 30 of 40 children (75\%) in the oralimmunotherapy group passed the oral food challenge of $10 \mathrm{~g}$ of egg-white powder (Table 2). The number of children in the oral-immunotherapy group who passed the oral food challenge increased after 10 months, even though the challenge dose was twice as high at 22 
months. The median cumulative dose that was successfully consumed by children in the oral-immunotherapy group during the oral food challenge at 22 months was $10.0 \mathrm{~g}$ (range, 1.5 to 10.0$)$.

Of the 30 children who passed the oral food challenge at 22 months, 29 underwent the oral food challenge at 24 months, and 11 passed. Thus, according to the intention-to-treat analysis, 11 of the 40 children (28\%) in the oral-immunotherapy group passed the oral food challenge at 24 months $(\mathrm{P}=0.03$, as compared with placebo) (Table 2$)$. Among the 18 children who underwent this challenge and did not pass, 5 consumed a dose of $7.5 \mathrm{~g}, 3 \mathrm{a}$ dose of $3.5 \mathrm{~g}, 5$ a dose of $1.5 \mathrm{~g}, 4$ a dose of $0.5 \mathrm{~g}$, and $1 \mathrm{a}$ dose of $0.1 \mathrm{~g}$. Since no children who received placebo passed the oral food challenge at 22 months, none were eligible to undergo the challenge at 24 months; none of the children in the placebo group met the primary end point.

Of the 22 children who were desensitized at 10 months, $9(41 \%)$ passed the oral food challenge at 24 months, as compared with 2 of 18 children (11\%) who were not desensitized $(\mathrm{P}=0.07)$. In the oral-immunotherapy group, 9 of the 18 children $(50 \%)$ who reached the maintenance dose of $2 \mathrm{~g}$ before 10 months passed the challenge at 24 months, as compared with 2 of the $22(9 \%)$ who did not reach the 2 -g dose before 10 months $(\mathrm{P}=0.006)$.

In the group of children who consumed egg ad libitum, no adverse events were reported at 30 months (11 children) or at 36 months ( 10 children). One participant was lost to follow-up after 30 months.

\section{IMMUNOLOGIC CORRELATES}

Baseline age, sex, and maximum dose on the first day were not predictive of sustained unresponsiveness. Several a priori, protocol-defined immune variables were evaluated to identify correlates of successful clinical outcomes over time (Table 3).

Median egg-specific IgG4 antibody levels at 10 months were higher in children who were desensitized at 10 months $(\mathrm{P}=0.007)$, those who were desensitized at 22 months $(\mathrm{P}=$ $0.005)$, and those who had sustained unresponsiveness at 24 months $(\mathrm{P}=0.02)$, as compared with children who did not pass the oral food challenge at these time points (Table 3, and Fig. $\mathrm{S} 1$ in the Supplementary Appendix). Similar correlations were found for changes from baseline in egg-specific IgG4 antibody levels (Fig. S2 in the Supplementary Appendix). Logistic-regression analysis confirmed these correlations, indicating that egg-specific IgG4 antibody levels at 10 months correlated with desensitization at 10 months and also predicted desensitization at 22 months and sustained unresponsiveness at 24 months.

At 10 months, egg-specific IgE antibody levels and basophil activation were lower in children who were successfully desensitized at 22 months than in those who were not $(\mathrm{P}=$ 0.02 and $\mathrm{P}=0.04$, respectively). However, these immune variables did not correlate with sustained unresponsiveness at 24 months.

The wheal size on skin-prick testing at 22 months was inversely associated with the likelihood of desensitization at 22 months $(\mathrm{P}=0.009)$ and with sustained unresponsiveness at 24 months $(\mathrm{P}=0.005)$ (Table 3, and Fig. S3 in the Supplementary Appendix). A decrease in wheal size from baseline to 22 months was also correlated with sustained unresponsiveness at 24 months $(\mathrm{P}=0.01)$ (Fig. S4 in the Supplementary Appendix). Logistic-regression analysis confirmed that a reduced wheal size at 22 months, as compared with baseline, correlated with sustained unresponsiveness at 24 months. 
As compared with children who received placebo, those who received oral immunotherapy had a decreased wheal size on skin-prick testing, reduced egg-induced basophil activation, and increased egg-specific IgG4 antibody levels over time, whereas no change in eggspecific IgE antibody levels was noted (Fig. S5 through S8 in the Supplementary Appendix).

\section{ADVERSE EVENTS}

All 55 children completed the initial-day dose escalation. Seven children (13\%) withdrew before the maintenance phase ( 2 in the placebo group and 5 in the oral-immunotherapy group). Of the 2 children in the placebo group who withdrew, 1 discontinued the study after completion of the initial dose escalation because of allergy-like symptoms and 1 because of transportation issues. Of the 5 children in the oral-immunotherapy group who withdrew within 5.5 months after starting therapy, 4 had allergic reactions, and 1 had an anxiety reaction. One additional child in the oral-immunotherapy group withdrew after the oral food challenge at 10 months but before the challenge at 22 months, owing to an allergic reaction associated with dosing (see the Supplementary Appendix).

Adverse events occurred most frequently in association with oral-immunotherapy dosing. The rates of adverse events were highest during the first 10 months of oral immunotherapy (Table 4). No severe adverse events occurred. Adverse events, most of which were oral or pharyngeal, were associated with $25.0 \%$ of 11,860 doses of oral immunotherapy with egg and $3.9 \%$ of 4018 doses of placebo. In the oral-immunotherapy group, $78 \%$ of children had oral or pharyngeal adverse events, as compared with $20 \%$ of those in the placebo group $(\mathrm{P}<0.001)$. After 10 months, the rate of symptoms in the oral-immunotherapy group decreased to $8.3 \%$ of 15,815 doses (data not shown). In addition to dosing-related symptoms, 437 other adverse events were reported; $96.0 \%$ were considered to be unrelated to dosing on the basis of the timing and the type of symptoms. All serious adverse events (three respiratory infections and one allergic reaction to peanuts) were considered to be unrelated to dosing.

\section{DISCUSSION}

Oral immunotherapy has previously been evaluated for its ability to desensitize persons to foods such as milk, peanuts, and eggs. ${ }^{14-20,26}$ The current study, unlike previous studies, enrolled a substantial number of children at multiple sites and showed sustained unresponsiveness in a double-blind, randomized, controlled study design with long-term follow-up during ad libitum consumption of the allergen. Two previous studies ${ }^{18,27}$ evaluating oral immunotherapy for milk and peanut allergies had double-blind, placebocontrolled designs, but they were smaller than the current study and neither involved more than two sites. A third study ${ }^{20}$ showed desensitization and sustained unresponsiveness after prolonged oral immunotherapy with milk but did not evaluate the ability of the participants to consume milk ad libitum. Desensitization alone is a therapeutically beneficial state because it confers protection against allergic reaction to accidental exposure. However, some participants in oral-immunotherapy studies who had only short-term desensitization subsequently had allergic symptoms after exposure to the suspect food during viral infections or after exercise. ${ }^{28}$ Sustained unresponsiveness, which occurred in $28 \%$ of the children in the current study, appears to be therapeutically more desirable than desensitization, in that the children had a higher threshold to the food allergen than would be expected according to the natural history, successfully incorporated egg into their diet, and were without symptoms at 36 months.

Suppression of mast-cell function, basophil activation, and modulation of lymphocyte responses are critical for the development of immune tolerance in response to allergen immunotherapy (e.g., subcutaneous immunotherapy). ${ }^{6,29,30}$ In the current study, the 
suppression of mast cells, as evidenced by decreased wheal size on skin-prick testing, and basophil activation were noted in children receiving oral immunotherapy, as compared with those receiving placebo, through 22 months and correlated with desirable clinical outcomes. Egg-specific IgG4 antibody levels at 10 months were increased by a factor of more than 100 above baseline values and correlated with sustained unresponsiveness. However, we identified no threshold elevations of egg-specific IgG4 antibody above the baseline value that were predictive of sustained unresponsiveness or that could be used as a substitute for the observed results of an oral food challenge to predict clinical outcomes. Increases in specific IgG4 antibody levels, with or without decreases in IgE antibody levels, have been associated with successful immunotherapy ${ }^{31-33}$ and loss of clinical sensitivity to milk and egg. ${ }^{18,20,34,35}$ After immunotherapy, the blocking activity present in serum is associated with allergen-specific IgG4 antibody, and it may be responsible for long-term immune tolerance after aeroallergen immunotherapy. 36,37

Although the results of the current study are consistent with the induction of sustained unresponsiveness, the data cannot formally exclude other possibilities. First, the children who passed the oral food challenge at 24 months might lose the sustained unresponsiveness if ad libitum egg consumption were discontinued. A more prolonged phase of egg avoidance after oral immunotherapy might have excluded this possibility, but it was considered impractical, owing to the difficulty in achieving compliance with longer-term avoidance of egg. Second, participants in the study might have spontaneously outgrown the egg allergy. This explanation is unlikely, given the inclusion criteria that predicted a low likelihood of outgrowing the egg allergy. ${ }^{21,22}$ Indeed, none of the children who received placebo passed the oral food challenge at 10 months, one did not pass the challenge at 22 months, and the others had persistently high levels of egg-specific IgE antibody at 22 months. Third, the children who passed the oral food challenge at 24 months might not have avoided consuming egg during the period of 4 to 6 weeks when they were not receiving oral immunotherapy, but we believe this to be unlikely.

In conclusion, we found that oral immunotherapy provides protection in a majority of children with egg allergy by raising the reaction threshold and represents a highly promising therapeutic intervention for food allergy. The approach is relatively safe in that reactions to dosing were mild (grade 1), with less than $1 \%$ of reactions scored as moderate (grade 2). However, some allergic reactions were of sufficient clinical significance that approximately $15 \%$ of the children who received oral immunotherapy did not complete the therapy, in most cases because of allergic reactions. The mechanisms underlying the success of oral immunotherapy and their relationship to natural immune tolerance are unknown. For oral immunotherapy to be recommended as a standard of care, it will be important to better define the risks of oral immunotherapy versus allergen avoidance, determine the dosing regimens with the most favorable outcomes, identify patients who are most likely to benefit from oral immunotherapy, and develop postdesensitization strategies ${ }^{38}$ that promote longterm immune tolerance.

\section{Supplementary Material}

Refer to Web version on PubMed Central for supplementary material.

\section{Acknowledgments}

Supported by grants from the National Institute of Allergy and Infectious Diseases (NIAID) (U19AI066738 and U01AI066560) and the National Institutes of Health-National Center for Research Resources Clinical Translational Science Awards and Clinical Research Centers (RR-024128, to Duke University Medical Center; RR-025005, to Johns Hopkins School of Medicine; RR-025780, to National Jewish Health; RR-029887, to Mount Sinai School of Medicine; and RR-029884, to the University of Arkansas for Medical Sciences). 
We thank D. Brown, M. Mishoe, J. Gau, S. Noone, M. Beksinska, J. Grabowska, K. Mudd, S. Driggers, P. Steele, J. Kamilaris, S. Carlisle, T. Hubbart, A. Hiegel, L. Christie, J. Straw, M. Groetch, J. Slinkard, J. Stone, S. Leung, K. Morgan, and K. Brown-Engelhardt for study coordination and support; the staff of the clinical research unit at each institution and the Statistical and Clinical Coordinating Center, without whose participation the study could not have been done; J. Poyser, for managing the project for CoFAR (NIAID); and the families who kindly participated.

\section{REFERENCES}

1. Branum AM, Lukacs SL. Food allergy among children in the United States. Pediatrics. 2009; 124:1549-1555. [PubMed: 19917585]

2. Sicherer SH, Noone SA, Muñoz-Furlong A. The impact of childhood food allergy on quality of life. Ann Allergy Asthma Immunol. 2001; 87:461-464. [PubMed: 11770692]

3. Eggesbø M, Botten G, Halvorsen R, Magnus P. The prevalence of allergy to egg: a populationbased study in young children. Allergy. 2001; 56:403-411. [PubMed: 11350303]

4. Bollinger ME, Dahlquist LM, Mudd K, Sonntag C, Dillinger L, McKenna K. The impact of food allergy on the daily activities of children and their families. Ann Allergy Asthma Immunol. 2006; 96:415-421. [PubMed: 16597075]

5. Brown HM. Would oral desensitization for peanut allergy be safer than avoidance? Ann Allergy Asthma Immunol. 2007; 98:203. [PubMed: 17304895]

6. Akdis CA, Akdis M. Mechanisms of allergen-specific immunotherapy. J Allergy Clin Immunol. 2011; 127:18-27. [PubMed: 21211639]

7. Frew AJ. Allergen immunotherapy. J Allergy Clin Immunol. 2010; 125(Suppl):S306-S313. [PubMed: 20176266]

8. Nelson HS, Lahr J, Rule R, Bock A, Leung D. Treatment of anaphylactic sensitivity to peanuts by immunotherapy with injections of aqueous peanut extract. J Allergy Clin Immunol. 1997; 99:744751. [PubMed: 9215240]

9. Oppenheimer JJ, Nelson HS, Bock SA, Christensen F, Leung DY. Treatment of peanut allergy with rush immunotherapy. J Allergy Clin Immunol. 1992; 90:256-262. [PubMed: 1500630]

10. Blumchen K, Ulbricht H, Staden U, et al. Oral peanut immunotherapy in children with peanut anaphylaxis. J Allergy Clin Immunol. 2010; 126(1):83.e1-91.e1. [PubMed: 20542324]

11. Buchanan AD, Green TD, Jones SM, et al. Egg oral immunotherapy in nonanaphylactic children with egg allergy. J Allergy Clin Immunol. 2007; 119:199-205. [PubMed: 17208602]

12. Burks AW, Jones SM. Egg oral immunotherapy in non-anaphylactic children with egg allergy: follow-up. J Allergy Clin Immunol. 2008; 121:270-271. [PubMed: 17889930]

13. Enrique E, Pineda F, Malek T, et al. Sublingual immunotherapy for hazelnut food allergy: a randomized, double-blind, placebo-controlled study with a standardized hazelnut extract. J Allergy Clin Immunol. 2005; 116:1073-1079. [PubMed: 16275379]

14. Jones SM, Pons L, Roberts JL, et al. Clinical efficacy and immune regulation with peanut oral immunotherapy. J Allergy Clin Immunol. 2009; 124:292-300. [PubMed: 19577283]

15. Longo G, Barbi E, Berti I, et al. Specific oral tolerance induction in children with very severe cow's milk-induced reactions. J Allergy Clin Immunol. 2008; 121:343-347. [PubMed: 18158176]

16. Meglio P, Bartone E, Plantamura M, Arabito E, Giampietro PG. A protocol for oral desensitization in children with IgE-mediated cow's milk allergy. Allergy. 2004; 59:980-987. [PubMed: 15291907]

17. Patriarca G, Nucera E, Roncallo C, et al. Oral desensitizing treatment in food allergy: clinical and immunological results. Aliment Pharmacol Ther. 2003; 17:459-465. [Erratum, Aliment Pharmacol Ther 2003;17:1205.]. [PubMed: 12562461]

18. Skripak JM, Nash SD, Rowley H, et al. A randomized, double-blind, placebo-controlled study of milk oral immunotherapy for cow's milk allergy. J Allergy Clin Immunol. 2008; 122:1154-1160. [PubMed: 18951617]

19. Staden U, Rolinck-Werninghaus C, Brewe F, Wahn U, Niggemann B, Beyer K. Specific oral tolerance induction in food allergy in children: efficacy and clinical patterns of reaction. Allergy. 2007; 62:1261-1269. [PubMed: 17919140] 
20. Keet CA, Frischmeyer-Guerrerio PA, Thyagarajan A, et al. The safety and efficacy of sublingual and oral immunotherapy for milk allergy. J Allergy Clin Immunol. 2012; 129:448-455. [PubMed: 22130425]

21. Sampson HA. Utility of food-specific IgE concentrations in predicting symptomatic food allergy. J Allergy Clin Immunol. 2001; 107:891-896. [PubMed: 11344358]

22. Savage JH, Matsui EC, Skripak JM, Wood RA. The natural history of egg allergy. J Allergy Clin Immunol. 2007; 120:1413-1417. [PubMed: 18073126]

23. Perry TT, Matsui EC, Conover-Walker MK, Wood RA. The relationship of allergen- specific IgE levels and oral food challenge outcome. J Allergy Clin Immunol. 2004; 114:144-149. [PubMed: 15241358]

24. Wanich N, Nowak-Wegrzyn A, Sampson HA, Shreffler WG. Allergen-specific basophil suppression associated with clinical tolerance in patients with milk allergy. J Allergy Clin Immunol. 2009; 123(4):789.e20-794.e20. [PubMed: 19348919]

25. Kunz B, Oranje AP, Labreze L, Stalder JF, Ring J, Taieb A. Clinical validation and guidelines for the SCORAD index: consensus report of the European Task Force on Atopic Dermatitis. Dermatology. 1997; 195:10-19. [PubMed: 9267730]

26. Nowak-Wegrzyn A, Sampson HA. Future therapies for food allergies. J Allergy Clin Immunol. 2011; 127:558-573. [PubMed: 21277625]

27. Varshney P, Jones SM, Scurlock AM, et al. A randomized controlled study of peanut oral immunotherapy: clinical desensitization and modulation of the allergic response. J Allergy Clin Immunol. 2011; 127:654-660. [PubMed: 21377034]

28. Varshney P, Steele PH, Vickery BP, et al. Adverse reactions during peanut oral immunotherapy home dosing. J Allergy Clin Immunol. 2009; 124:1351-1352. [PubMed: 19913285]

29. Akdis M. Immune tolerance in allergy. Curr Opin Immunol. 2009; 21:700-707. [PubMed: 19700272]

30. Akdis M, Akdis CA. Mechanisms of allergen-specific immunotherapy. J Allergy Clin Immunol. 2007; 119:780-791. [PubMed: 17321578]

31. Francis JN, James LK, Paraskevopoulos G, et al. Grass pollen immunotherapy: IL-10 induction and suppression of late responses precedes IgG4 inhibitory antibody activity. J Allergy Clin Immunol. 2008; 121(5):1120.e2-1125.e2. [PubMed: 18374405]

32. Scadding GW, Shamji MH, Jacobson MR, et al. Sublingual grass pollen immunotherapy is associated with increases in sublingual Foxp3-expressing cells and elevated allergen-specific immunoglobulin G4, immunoglobulin A and serum inhibitory activity for immunoglobulin Efacilitated allergen binding to B cells. Clin Exp Allergy. 2010; 40:598-606. [PubMed: 20184605]

33. Clements JL, Yang B, Ross-Barta SE, et al. Requirement for the leukocyte-specific adapter protein SLP-76 for normal T cell development. Science. 1998; 281:416-419. [PubMed: 9665885]

34. James JM, Sampson HA. Immunologic changes associated with the development of tolerance in children with cow milk allergy. J Pediatr. 1992; 121:371-377. [PubMed: 1517910]

35. Lemon-Mule H, Sampson HA, Sicherer SH, Shreffler WG, Noone S, Nowak-Wegrzyn A. Immunologic changes in children with egg allergy ingesting extensively heated egg. J Allergy Clin Immunol. 2008; 122(5):977.e1-983.e1. [PubMed: 18851876]

36. James LK, Shamji MH, Walker SM, et al. Long-term tolerance after allergen immunotherapy is accompanied by selective persistence of blocking antibodies. J Allergy Clin Immunol. 2011; 127(2):509.e1-5-516.e1-5. [PubMed: 21281875]

37. Shamji MH, Durham SR. Mechanisms of immunotherapy to aeroallergens. Clin Exp Allergy. 2011; 41:1235-1246. [PubMed: 21762223]

38. Golden DB, Kagey-Sobotka A, Lichtenstein LM. Survey of patients after discontinuing venom immunotherapy. J Allergy Clin Immunol. 2000; 105:385-390. [PubMed: 10669863] 


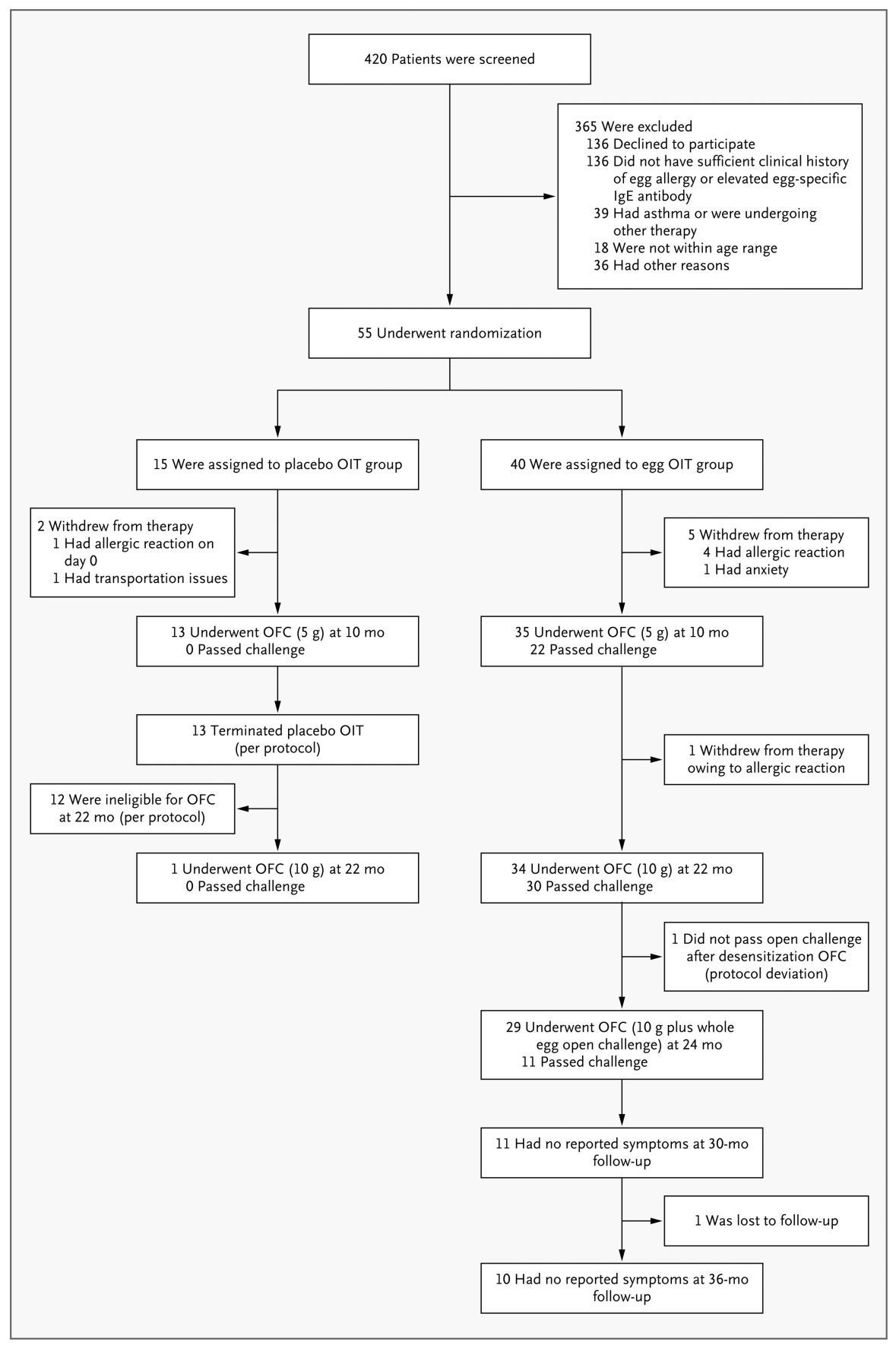

Figure 1. Study Enrollment, Randomization, and Outcomes

Eligibility criteria included both a convincing clinical history of egg allergy and elevated levels of egg-specific IgE antibody. No oral food challenge (OFC) was performed at baseline. The 55 children who met the screening requirements were randomly assigned to receive placebo or oral immunotherapy (OIT) with egg. After the challenge at 10 months, the study was unblinded, and all children who had received placebo were followed longitudinally without further dosing. Children in the placebo group were not eligible for the challenge at 22 months unless the level of egg-specific IgE antibody was less than $2 \mathrm{kU}$ per liter. All children in the OIT group continued to receive OIT after the challenge at 10 months, until the challenge at 22 months. Of the 30 children who passed the challenge at 22 
months, 29 stopped receiving OIT for 4 to 6 weeks and then underwent a challenge to assess sustained unresponsiveness at 24 months. All 11 children who passed the challenge at 24 months were placed on an ad libitum egg diet, with subsequent evaluation at 30 months (11 children) and 36 months (10 children). 
Table 1

Baseline Characteristics and Dosing Responses, According to Study Group. *

\begin{tabular}{|c|c|c|}
\hline Characteristic & $\begin{array}{l}\text { Placebo } \\
(\mathbf{N}=15)\end{array}$ & $\begin{array}{c}\text { Oral Immunotherapy } \\
\text { with Egg } \\
(\mathbf{N}=40)\end{array}$ \\
\hline \multicolumn{3}{|l|}{ Age $-\mathrm{yr}$} \\
\hline Median & 7.0 & 7.0 \\
\hline Range & $5.0-10.0$ & $5.0-11.0$ \\
\hline \multicolumn{3}{|l|}{ Atopic dermatitis score ${ }^{\dagger}$} \\
\hline Median & 3.0 & 3.5 \\
\hline Range & $0.0-8.0$ & $0.0-7.0$ \\
\hline \multicolumn{3}{|l|}{ Total IgE antibody — kU/liter } \\
\hline Median & 1060.9 & 1095.5 \\
\hline Range & $137.2-3440.9$ & $38.3-10,097.0$ \\
\hline \multicolumn{3}{|c|}{ Egg-specific IgE antibody — kU/liter } \\
\hline Median & 25.7 & 10.3 \\
\hline Range & $4.1-89.0$ & $3.7-231.1$ \\
\hline \multicolumn{3}{|c|}{ Egg-specific IgG4 antibody — mg/liter } \\
\hline Median & 0.3 & 0.2 \\
\hline Range & $0.0-9.7$ & $0.0-10.8$ \\
\hline \multicolumn{3}{|c|}{ Wheal diameter on skin-prick testing with egg $-\mathrm{mm}$} \\
\hline Median & 13.0 & 10.5 \\
\hline Range & $7.5-20.0$ & $2.5-26.0$ \\
\hline \multicolumn{3}{|c|}{ Age at first allergic reaction to egg $-\mathrm{yr}$} \\
\hline Median & 1.0 & 1.0 \\
\hline Range & $0.0-5.0$ & $0.0-8.0$ \\
\hline \multicolumn{3}{|c|}{ Maximum initial-day escalation dose $-\mathrm{mg}$} \\
\hline Median & $50.0^{*}$ & 18.5 \\
\hline Range & $50.0-50.0$ & $6.0-50.0$ \\
\hline Initial build-up dose $-\mathrm{mg}$ & & \\
\hline
\end{tabular}




\begin{tabular}{|lcc|}
\hline Characteristic & $\begin{array}{c}\text { Placebo } \\
(\mathbf{N}=\mathbf{1 5})\end{array}$ & $\begin{array}{c}\text { Oral Immunotherapy } \\
\text { with Egg } \\
(\mathbf{N}=\mathbf{4 0})\end{array}$ \\
\hline Median & $50.0^{.}$ & 9.0 \\
\hline Range & $12.0-50.0^{\xi}$ & $3.0-50.0$ \\
\hline Children who consumed a 2-g maintenance dose - no. (\%) & & \\
\hline Before oral food challenge at $10 \mathrm{mo}$ & $12(80)^{\frac{F}{*}}$ & $18(45)$ \\
\hline Before oral food challenge at $22 \mathrm{mo}$ & $\mathrm{NA}$ & $33(82)$ \\
\hline
\end{tabular}

There were no significant differences between the placebo group and the oral-immunotherapy group. NA denotes not applicable.

${ }^{\dagger}$ Scores for atopic dermatitis range from 0 to 9 , with 0 indicating no disease and 9 the most severe disease state. ${ }^{25}$

$t^{*}$ The doses for placebo are in milligrams of placebo powder.

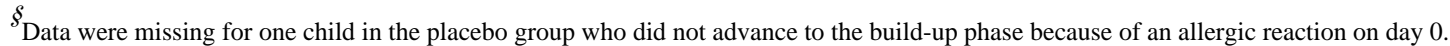


Table 2

Success Rates on Oral Food Challenge.

\begin{tabular}{|c|c|c|c|c|c|}
\hline \multirow[t]{3}{*}{ Challenge } & \multicolumn{2}{|c|}{ Participants Tested ${ }^{*}$} & \multicolumn{2}{|r|}{ Response Rate } & \multirow{3}{*}{ P Value } \\
\hline & $\begin{array}{r}\text { Placebo } \\
(\mathbf{N}=15)\end{array}$ & $\begin{array}{l}\text { Oral Immunotherapy } \\
\qquad(\mathbf{N}=\mathbf{4 0})\end{array}$ & $\begin{array}{l}\text { Placebo } \\
(\mathrm{N}=15)\end{array}$ & $\begin{array}{l}\text { Oral Immunotherapy } \\
\qquad(\mathbf{N}=\mathbf{4 0})\end{array}$ & \\
\hline & \multicolumn{2}{|r|}{ number } & \multicolumn{2}{|c|}{ number (percent) } & \\
\hline Desensitization, $5 \mathrm{~g}$ at $10 \mathrm{mo}$ & 13 & 35 & 0 & $22(55)$ & $<0.001$ \\
\hline Desensitization, $10 \mathrm{~g}$ at $22 \mathrm{mo}$ & $1^{\dagger}$ & 34 & 0 & $30(75)$ & $<0.001$ \\
\hline Sustained unresponsiveness at $24 \mathrm{mo}^{f}$ & 0 & $29 \xi$ & 0 & $11(28)$ & 0.03 \\
\hline
\end{tabular}

* Oral food challenges were not performed in children who withdrew from the study owing to dose-limiting symptoms. Thus, the actual number of oral food challenges performed is provided.

$\dagger^{\prime}$

At 22 months, children who received placebo did not undergo another oral food challenge, given a history of not passing an oral food challenge within the past year, unless the level of antibody declined to less than $2 \mathrm{kU}$ per liter; this decline occurred in only 1 child.

${ }^{*}$ The oral food challenge for sustained unresponsiveness comprised $10 \mathrm{~g}$ of egg-white powder and a whole cooked egg. The $95 \%$ confidence interval for the between-group difference in the response rate was $4.3 \%$ to $43.9 \%$.

$\xi_{\text {Although }} 30$ children initially passed the oral food challenge at 22 months, 1 also underwent a challenge with a whole egg (protocol deviation) and did not pass, so only 29 underwent the oral food challenge for sustained unresponsiveness. 


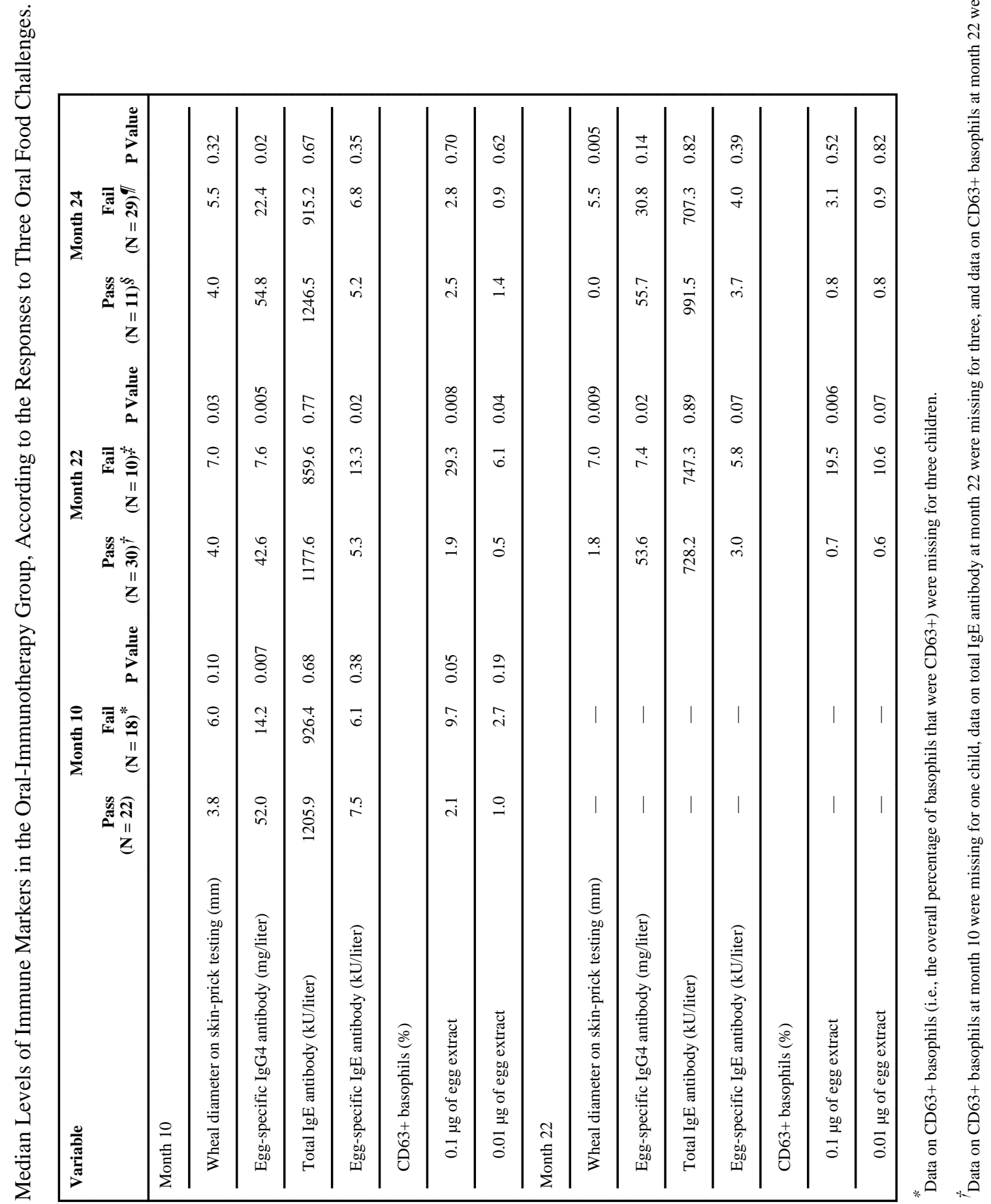




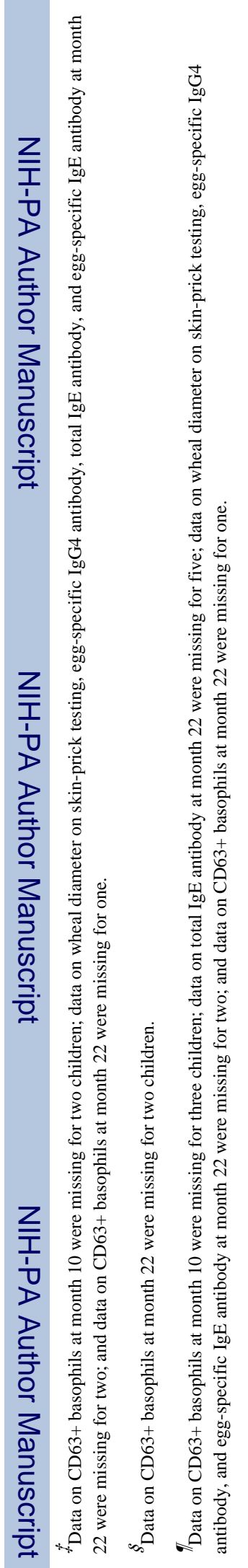




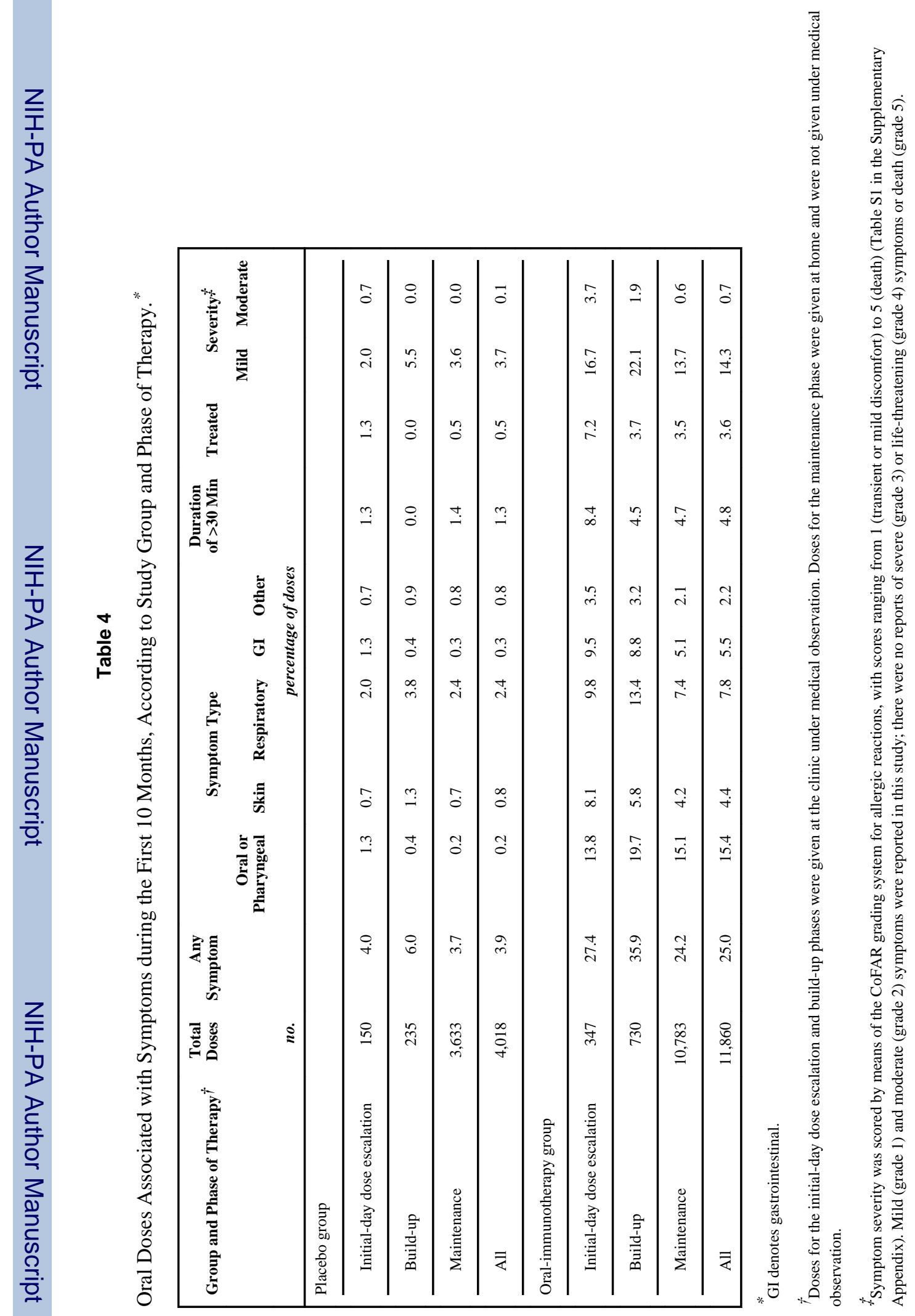

NEngl J Med. Author manuscript; available in PMC 2013 January 19. 